\title{
Hennig in English: hypotheses for entomology's systematists
}

\section{R. A. Crowson}

Insect Phylogeny. By W. Hennig. Translated and adapted by A. C. Pont, with revisionary notes by $D$. Schlee. Pp.514. ISBN 0-471-27848-3. (Wiley: 1981.) £28, \$84.

Will., Hennig's 1969 volume, Die Phylogenie der Insekten (published by Waldemar Kramer), while undoubtedly a work of scholarly importance, received rather less than due attention in the English-speaking world. At the time of his death in 1976, Hennig was accumulating notes for a revised English-language version of the work. In its lieu, the present volume provides a translation of the 1969 text into good and readable English, interspersed with Hennig's surviving notes for a revision, and supplementary notes by younger, mainly German entomologists designed to bring into consideration work published since 1968. The bibliography now includes some references as recent as 1980.

The "methodological introduction" summarizes Hennig's phylogenetic methods, more fully set out in his Phylogenetic Systematics (University of Illinois Press, 1966). It is followed by a review of Palaeozoic and Mesozoic fossil data on insects, a systematic account of the phylogenetic relations of modern insects down to about order or suborder level, and a more detailed review of the phylogenetic relations of Mesozoic insects. Illustrations are mainly dendrograms or pictures of wings; the bibliography is extensive though far from exhaustive.

The main phylogenetic conclusions, not significantly changed by the later annotations, agree largely with those of Boudreaux's Arthropod Phylogeny with

\section{Forms of magnetic behaviour}

\section{B. R. Coles}

Introduction to the Magnetic Properties of Solids. By A.S. Chakravarty. Pp.696. ISBN 0-471-07737-2. (Wiley: 1981.) £35, $\$ 81$.

MAGNETISM continues to present challenges to our physical insight and abilities to produce firmly based formal theories. With its wide range of phenomena and richness in relevant concepts (spin, exchange, spin-waves, domains, ferromagnetism, spin density waves, crystal fields and so on), it is a much more difficult subject to present to the student in an orderly fashion than, on the one hand, classical electricity and magnetism or, on the other, quantum mechanics. Faced with this dilemma authors either accept the complexity and diversity of the real world of the magnetic properties of matter or turn their back on it until they have laid the formal theoretical foundations which they are convinced will ultimately be the basis of explanations of the behaviour of all magnetic matter. As this book (which is firmly in the second category) shows, the difficulty of the latter approach is that the materials and properties described tend less to be those of intrinsic interest than those that illustrate the bits of theory that can be tackled fairly rigorously. It contains very few figures showing the behaviour of real materials (the first is on p.150 and the second on p.265) and those are chosen solely to compare with rather specific calculations rather than because of their power to illustrate important effects.

For the theoretical physicist who seeks interesting problems that give promise of possible solutions with existing techniques, this will be a helpful introduction that moulds his thinking along the lines that have been thoroughly explored and found useful. It does not, however, give the introduction promised in the title to the magnetic properties of real solids in all their diversity or even to some modern theoretical developments. Some omissions are understandable, given the tenor of the book, but it is strange to find a transition from an account of the band theory of solids to theories of itinerant ferromagnetism - which oddly fails to refer the student to Herring's classic text (Magnetism edited by $\mathrm{G}$. Rado and $\mathrm{H}$. Suhl, Vol IV; Academic Press, 1972) with no reference to the susceptibilities of metals that do not become magnetically ordered. The treatment of spin-wave spectra is surprisingly brief and there is no discussion of the powers of neutron scattering in their investigation. There is also no discussion of the phase transition aspects of magnetism which are currently a focus of much theoretical effort "critical phenomena" and "renormalization group" do not appear in the index. It is a pity that space that might have introduced even these theoretical aspects was taken up with extended treatments of standard background theoretical formalism which are easily found elsewhere.

B. R. Coles is Professor of Solid State Physics at Imperial College, University of London.
Special Reference to Insecta (Wiley, 1979) who also applied Hennigian methods, though Hennig goes into more detail. A notable feature is the use of a numerical system denoting taxa, based on Hennig's belief that phyletic splittings are almost always binary, these numbers being directly convertible into a topologically correct dendrogram. One effect of the binary system is the use of a large number of classificatory levels - thus it takes eight splittings to get from Insecta (= Hexapoda) to an order such as Diptera, though only two to Diplura.

Perhaps the most important of the additions are due to Dieter Schlee, who brings into consideration a good deal of recently described fossil evidence, not known to Hennig in 1969. Otherwise, the later annotators seem mainly concerned with promulgating their own views about particular orders, not always in a very scholarly manner. Some of these writers, and even Hennig himself, may at times be accused of unduly dogmatic assertion thus Hennig states that the theory of a blood-sucking ancestor for Diptera "is certainly not correct"; Mickoleit writes "There is no longer any doubt that the Mecoptera are a monophyletic group"; and Kinzelbach states flatly that "Strepsiptera have no evident gula". Hennig himself, and M. Baehr, are both guilty of misunderstanding and misrepresenting my own views on the Coleoptera at some points.

For the non-entomological systematist, the work should be of interest as the example of Hennig's own application of his method to higher-level classification of a major section of the animal kingdom. The data used in this work are drawn almost entirely from the fields of classical comparative anatomy and palaeontology; its conclusions should be taken as scientific hypotheses, which could be tested against evidence from fields such as "macromolecular systematics" not taken into account by Hennig and his coworkers.

It is to be regretted that neither Hennig nor his annotators have tried seriously to establish ancestral modes of life for the taxa with which they are concerned. The result is that the book has little of interest to the new, rising school of palaeoecologists, or even to palaeogeographers, despite Hennig's own interest in geographical distribution as evidence for phylogeny. The main users of the book will undoubtedly be entomologists and palaeoentomologists, for whom this well produced volume should be a desirable acquisition.

R. A. Crowson is a Senior Lecturer in the Zoology Department at the University of Glasgow. 